\title{
Scientific Issues for Assessing Biosimilars in the United States
}

\section{Shein-Chung Chow*}

Duke University School of Medicine, Durham, North Carolina, USA

In the United States (US), for small molecule drug products, when an innovative (brand-name) drug product is going off patent, pharmaceutical and/or generic companies may file an Abbreviated New Drug Application (ANDA) for approval of the generic copies of the brand-name drug. In 1984, the US Food and Drug Administration (FDA) was authorized to approve generic drug products under the Drug Price Competition and Patent Term Restoration Act, which is also known as the Hatch and Waxman Act. For approval of generic (small molecule) drug products, the FDA requires that evidence in average of bioavailability, which is measured in terms of the rate and extent of drug absorption, be provided through the conduct of bioequivalence studies [1]. For small molecule generics, reductions in price of around $80 \%$ have been observed after the first six months to a year of generics entry to the market in countries such as Germany, UK and the US $[2,3]$.

Unlike small molecule drug products, the generic versions of biologic products are similar biological drug products (SBDP). The SBDP are not generic drug products (drug products with identical active ingredient(s) as the innovative drug product). The concept for development of SBDP, which are made of living cells, is very different from that of the small molecule generic drug products. The SBDP are usually referred to as biosimilars by European Medicines Agency (EMA) of European Union (EU), follow-on biologics (FOB) by the US FDA, and subsequent entered biologics (SEB) by the Public Health Agency (PHA) of Canada. EMA has been successful in devising a system for authorising the marketing of biosimilar products and 14 biosimilars are currently on the market in the major countries of the EU [4]. Unlike small molecule generic drug products, the price difference between biosimilars and originator biological products is likely to be smaller than that observed between originator and generics, given that biosimilars incur higher research and development costs. Generally, biosimilars are priced about $30 \%$ less than the originator product.

In the past couple year, regulatory approval pathway for assessment of biosimilars of biological products has received much attention in the United States. In practice, standard methods for assessment of bioequivalence for regulatory approval of generic drug products are usually applied to assessment of biosimilars. This, however, is not appropriate due to some fundamental differences between small molecule generic drug products and (large molecule) biosimilar products [5]. For example, biosimilar products are made of living cells and have heterogeneous structure (usually mixtures of related molecules) which is difficult to characterize. In addition, biosimilar products are often variable and sensitive to environmental conditions such as light and temperature. A small change could result in a drastic change in clinical outcomes.

On March 23, 2010, the Biologics Price Competition and Innovation (BPCI) Act (as part of the Affordable Care Act) was passed by the US Congress which gives the FDA the authority to approve similar biological drug products (biosimilars). As indicated in the BPCI Act, a biosimilar product is defined as a product that is highly similar to the reference product notwithstanding minor differences in clinically inactive components and there are no clinically meaningful differences in terms of safety, purity, and potency. Based on this definition, we would interpret that a biological medicine is biosimilar to a reference biological medicine if it is highly similar to the reference in safety, purity, and efficacy. However, little or no discussion regarding how similar is considered highly similar in the BPCI Act. As indicated in the Subsection (a) (2) amends the Public Health Act Subsection $351(\mathrm{k})(3)$, a biological product is considered to be interchangeable with the reference product if (i) the biological product is biosimilar to the reference product; and (ii) it can be expected to produce the same clinical result in any given patient. In addition, for a biological product that is administered more than once to an individual, the risk in terms of safety or diminished efficacy of alternating or switching between use of the biological product and the reference product is not greater than the risk of using the reference product without such alternation or switch. Thus, there is a clear distinction between biosimilarity and interchangeability. In other words, biosimilarity does not imply interchangeability, which is much more stringent. Intuitively, if a test product is judged to be interchangeable with the reference product then it may be substituted, even alternated, without a possible intervention, or even notification, of the health care provider. However, the interchangeability is expected to produce the same clinical result in any given patient, which can be interpreted as that the same clinical result can be expected in every single patient. In reality, conceivably, lawsuits may be filed if adverse effects are recorded in a patient after switching from one product to another.

Following the passage of the BPCI Act, in order to obtain input on specific issues and challenges associated with the implementation of the BPCI Act, the US FDA conducted a two-day public hearing on Approval Pathway for Biosimilar and Interchangeability Biological Products held on November 2-3, 2010 at the FDA in Silver Spring, Maryland, USA. Several scientific factors were raised and discussed at the public hearing. These scientific factors include, but are not limited to, the definition of "highly similar" including the degree of similarity, criteria for assessing biosimilarity (e.g., average versus variability and moment-based versus probability-based criteria), study design (e.g., crossover versus parallel design), statistical methods (e.g., the potential use of Bayesian approach), and tests for comparability in quality attributes of manufacturing process and/or immunogenicity [6]. These issues primarily focus on the assessment of biosimilarity. The issue of interchangeability in terms of the concepts of alternating and switching were also mentioned and discussed. These scientific factors have generated a lot of research interest. As a result, one of the leading biostatistics journals, Statistics in Medicine, has decided to publish a special issue on biosimilars (guest co-editors: Yi Tsong of FDA and

${ }^{*}$ Corresponding author: Shein-Chung Chow, PhD, Professor, Department of Biostatistics and Bioinformatics, Duke University School of Medicine, 2424 Erwin Road, Hock Suite 1102, Room 11068, Durham, NC 27710, USA. E-mail: sheinchung.chow@duke.edu

Received April 09, 2012; Accepted April 09, 2012; Published April 11, 2012

Citation: Chow SC (2012) Scientific Issues for Assessing Biosimilars in the United States. J Biomet Biostat 3:e107. doi:10.4172/2155-6180.1000e107

Copyright: ( 92012 Chow SC. This is an open-access article distributed under the terms of the Creative Commons Attribution License, which permits unrestricted use, distribution, and reproduction in any medium, provided the original author and source are credited. 
Shein-Chung Chow of Duke University School of Medicine; to be published in late 2012 or early 2013) attempting to address some of these scientific issues. However, many of these scientific factors still remain unresolved and more research on these topics is definitely needed.

On February 9, 2012, the U.S. Food and Drug Administration (FDA) released three draft guidances about demonstrations of biosimilarity. These draft guidances are (i) Scientific Considerations in Demonstrating Biosimilarity to a Reference Product, (ii) Quality Considerations in Demonstrating Biosimilarity to a Reference Protein Product, (iii) Biosimilars: Questions and Answers Regarding Implementation of the Biologics Price Competition and Innovation (BPCI) Act of 2009. As stated, these guidances are not only (i) intended to assist sponsors in demonstrating that a proposed therapeutic protein product is biosimilar to a reference product for purpose of the submission of a marketing application under section 351(k) for the Public Health Service Act, but also (ii) to describe the FDA's current thinking on factors to consider when demonstrating that a proposed protein product is highly similar to a reference product licensed under section 351(a) of the PHS Act for purpose of submitting a marketing application under section $351(\mathrm{k})$ of the PHS Act. In addition, the guidances provide answers to common questions from sponsors interested in developing proposed biosimilar products; biologics license application (BLA) holders, and other interested parties regarding FDA's interpretation of the Biologics Price Competition and Innovation (BPCI) Act of 2009.

In the draft guidances, FDA suggests the use of stepwise approach for assessing biosimilarity. The stepwise approach is to evaluate biosimilarity step by step. The idea is well understood. However, little or no information regarding criteria for assessment of biosimilarity to be used at each step was mentioned. Some scientific issues regarding the suggested stepwise approach are raised. These issues include (i) How many steps are required? (ii) Does the order of the steps matter? (iii) Should each step carry the same weight (in other words, are some steps more important than others)? and (iv) How to control the overall type I error rate? These issues need to be addressed for feasibility and validity of the stepwise approach. In the draft guidance, FDA also introduces the concept of totality-of-the-evidence. The FDA seems to suggest a scoring system for measuring the totality-of-the-evidence in order to account for (i) the distinction between local biosimilarity and global biosimilarity, (ii) degree of biosimilarity which may vary from domain to domain, and (iii) each domain may carry different weights. To address the totality-of-the-evidence, the following totality biosimilarity index derived based on biosimilarity index from each domain proposed by Chow [7] and Chow et al. [8] may be useful. At each domain, biosimilarity index can be obtained by the following steps:

Step 1: Assess average biosimilarity based on a given criterion, e.g. $(80 \%, 125 \%)$ based on $\log$ -

\section{transformed data;}

Step 2: Calculate the biosimilarity index (i.e., reproducibility) based on the observed ratio and variability;

Step 3: Claim biosimilarity if the $95 \%$ confidence lower bound of the biosimilarity index is larger than $\mathrm{p}_{0}$, a pre-specified number.

Note that in practice, $\mathrm{p}_{0}$ can be obtained based on an estimated of reproducibility probability of a study comparing a reference product to itself (the reference product). We will refer to such a study as an R-R study [7]. Similarly, the totality biosimilarity index across domains can be obtained as follows:

Step 1: Obtain $\mathrm{p}_{\mathrm{i}}$, the biosimilarity index for the ith domain,
Step 2: Define the totality biosimilarity indexas $\mathrm{P}_{\mathrm{T}}=\sum_{\mathrm{i}=1}^{\mathrm{K}} \mathrm{w}_{\mathrm{i}} \mathrm{p}_{\mathrm{i}}$, where $\mathrm{w}_{\mathrm{i}}$ is the weight for the $\mathrm{i}^{\text {th }}$ domain, where $\mathrm{i}=1, \ldots, \mathrm{K}$ (number of domains);

Step 3: Claim biosimilarity if the $95 \%$ confidence lower bound of $\mathrm{p}_{\mathrm{T}}$ is greater than a pre-specified value $\mathrm{p}_{\text {T0 }}$.

Similar to biosimilarity index, $\mathrm{p}_{\mathrm{T} 0}$ can be determined based on an estimated of totality biosimilarity index for studies comparing a reference product to itself (the reference product). The above described biosimilarity index or totality biosimilarity index has the advantages that (i) it is robust with respect to the selected study endpoint, biosimilarity criteria, and study design, (ii) it takes variability into consideration (one of the major criticisms in the assessment of average bioequivalence), (iii) it allows the definition and assessment the degree of similarity (in other words, it provides partial answer to the question that "how similar is considered similar?" and (iv) the use of biosimilarity index or totality biosimilarity index will reflect the sensitivity of heterogeneity in variance.

In the current FDA draft guidance, little or no information regarding interchangeability was mentioned. Unlike drug interchangeability in terms of prescribability and switch ability [1], the FDA has slightly different perception of interchangeability for biosimilars. From the FDA's perspectives, interchangeability includes the concept of switching and alternating between an innovative biologic product $(\mathrm{R})$ and its follow-on biologics (T). The concept of switching is referred to as not only the switch from "R to T" or "T to R" (narrow sense of switch ability), but also "T to T" and "R to R" (broader sense of switch ability). As a result, in order to assess switching, biosimilarity for " $R$ to T", "T to R", "T to T", and " $R$ to R" need to be assessed based on some biosimilarity criteria under a valid study design. On the other hand, the concept of alternating is referred to as either the switch from $\mathrm{T}$ to $\mathrm{R}$ and then switch back to $\mathrm{T}$ (i.e., "T to $\mathrm{R}$ to $\mathrm{T}$ ") or the switch from $\mathrm{R}$ to $\mathrm{T}$ and then switch back to $\mathrm{R}$ (i.e., "R to $\mathrm{T}$ to $\mathrm{R}$ ". Thus, the difference between "the switch from $\mathrm{T}$ to $\mathrm{R}$ " or "the switch from $\mathrm{R}$ to $\mathrm{T}$ " and "the switch from $\mathrm{R}$ to $\mathrm{T}$ " or "the switch from $\mathrm{T}$ to $\mathrm{R}$ " needs to be assessed for addressing the concept of alternating. For assessment of bioequivalence for chemical drug products, a standard two-sequence, two-period $(2 \times 2)$ crossover design is often considered, except for drug products with relatively long half-lives. Since most biosimilar products have relatively long half-lives, it is suggested that a parallel group design should be considered. However, parallel group design does not provide independent estimates of variance components such as interand intra-subject variabilities and variability due to subject-by-product interaction. Thus, it is a major challenge for assessing biosimilars under parallel group designs. In order to assess biosimilarity for " $\mathrm{R}$ to $\mathrm{T}$ ", "T to R", "T to T", and " $\mathrm{R}$ to R", the Balaam's $4 \times 2$ crossover design, i.e., (TT, RR, TR, RT) may be useful. For addressing the concept of alternating, a two-sequence, three-period dual design, i.e., (TRT, RTR) may be useful. For addressing both concepts of switching and alternating for drug interchangeability of biosimilars, a modified Balaam's crossover design, i.e., (TT, RR, TRT, RTR) is then recommended.

In summary, according to the definitions given in the BPCI Act, there is a clear distinction between biosimilarity and Interchangeability. Although the recent FDA draft guidances did provide some insights regarding the assessment of biosimilarity of biosimilars, many scientific issues such as the definition of highly similar, criteria for biosimilarity, the feasibility of the stepwise approach, statistical tests for comparability in quality attributes, and the assessment of totality-of-the-evidence still remain unresolved. Regarding drug interchangeability, in practice, it is difficult, if not impossible, to demonstrate that the expected 
"same clinical result in any given patient". However, it is possible to demonstrate "same clinical result in any given patient with certain assurance“. Under a given study design, the biosimilarity index and/ or totality biosimilarity index proposed by Chow [7] and Chow et al. [9] may be considered to develop alternating index and/or switching index for addressing interchangeability in terms of alternating and/or switching. However, further research is necessary. It should be noted that in order to obtain input and comments of the draft guidances and to discuss the issue of interchangeability, FDA will host a public hearing at FDA, Silver Spring, Maryland, on May 11, 2012. It is our hope that more specific guidances that cover design and analysis for assessment of biosimilars and discussion of the unresolved scientific issues would be developed for implementation of BPCI Act.

\section{References}

1. Chow SC, Liu JP (2008) Design and Analysis of Bioavailability and Bioequivalence Studies- Revised and Expanded. Third Edition, Taylor \& Francis, New York.
2. Simoens S, Verbeken G, Huys I (2011) Market access of biosimilars: not only a cost issue. Oncologie 13: 218-221.

3. GaBI Online (2012a) Generics and Biosimilars Initiative. Generics grab $80 \%$ share of US market and fill $78 \%$ of prescriptions, Mol, Belgium.

4. GaBI Online (2011b).Generics and Biosimilars Initiative. Biosimilars approved in Europe, Mol, Belgium.

5. Chow SC, Liu JP (2010) Statistical assessment of biosimilar products. Journal of Biopharmaceutical Statistics 20: 10-30.

6. Chow SC, Hsieh E, Chi E, Yang J (2010) A comparison of moment-based and probability based criteria for assessment of follow-on biologics. J Biopharm Stat 20: $31-45$.

7. Chow SC (2011) Quantitative evaluation of bioequivalence/biosimilarity. J Bioequiv Availab S1: 1-8.

8. Chow SC, Endrenyi L, Lachenbruch PA, Yang LY, Chi E (2011) Scientific factors for assessing biosimilarity and drug Interchangeability of follow-on Biologics. Biosimilars 1: 13-26. 